\title{
A single-nucleotide polymorphism involved in alternative splicing of PTEN is associated with chemotherapy resistance in breast cancer in Chinese population
}

Jing Wang

Lanzhou University

Shasha Zhang

Lanzhou University

Zhongliang Zhang

First People's Hospital of Lanzhou City

Qinglong Ma

Lanzhou University

Xiaohua Chen

Chinese Academy of Sciences

Dapeng Zhao

Chinese Academy of Sciences

Meie Zhao

Lanzhou University

Chunwei Wang

Gansu Provincial Hospital

Cuixia Di

Chinese Academy of Sciences

xiaodong xie ( $\boldsymbol{\sigma}$ xdxie@lzu.edu.cn )

Lanzhou University

Research

Keywords: PTEN, single nucleotide polymorphism, chemotherapy resistance, alternative splicing, breast cancer, silico analysis

Posted Date: April 6th, 2021

DOl: https://doi.org/10.21203/rs.3.rs-360619/v1 
License: (c) (i) This work is licensed under a Creative Commons Attribution 4.0 International License. Read Full License 


\section{Abstract \\ Background}

Chemoresistance is still the main reason for the failure of breast cancer treatment and is the main cause of death of breast cancer patients. Although many studies have shown the association between genetic polymorphisms of PTEN and chemotherapy resistance, the molecular mechanism of breast cancer chemotherapy has not been further studied. This study aims to investigate the potential association between PTEN gene polymorphism and breast chemotherapy resistance in the Chinese population, and explore whether alternative splicing of the PTEN gene is affected by the gene polymorphism.

\section{Methods}

The study included 234 patients with breast cancer chemotherapy, 157 chemotherapy sensitive cases and 77 chemotherapy resistant cases. rs786204926 of the PTEN gene was analysed by Sanger sequence and Sequenom MassArray typing technology. Furthermore, we used silicon analysis to predict whether polymorphism affect the process of alternative splicing and to analyze how it affects.

\section{Results}

In genotyping and allelic analysis, there was a significant association between rs786204926 polymorphism and breast cancer chemotherapy resistance. Carrying the $\mathrm{G}$ allele or AG genotype will reduce the risk of breast cancer-based resistance to chemotherapy with anthracyclines. Silicon analysis showed that the mutation of rs786204926 produced a new receptor site, which might affect alternative splicing of PTEN gene.

\section{Conclusions}

We speculate that the mechanism of breast cancer chemotherapy resistance might be caused by a change in the alternative splicing caused by the rs 786204926 of the PTEN gene. Thus, our study might provide theoretical guidance for the individualized treatment of clinical breast cancer patients.

\section{Background}

Breast cancer is a malignant tumor that occurs in breast epithelial tissue and is the most common malignant tumor in women[1]. Breast cancer has become a serious health problem threatening women all over the world[2, 3]. Although chemotherapy is one of the main treatments for breast cancer, chemotherapy resistance is still the main reason for the failure of breast cancer treatments $[4,5]$. Therefore, the identification of genetic mechanisms involved in chemotherapy resistance is crucial for predicting the drug response of tumor with genetic mutations[6, 7]. Studies have reported that the key 
mutation of the tumor suppressor gene might be the main factor of drug resistance in the treatment for cancer patients[8-10].

Currently, there are many tumor suppressor genes involved in breast cancer chemotherapy resistance, including MDR1, ABCG2 and PTEN [11-13]. The PTEN gene is one of the most common inactivated tumor suppressor genes[14]. Many studies have reported that PTEN is associated with resistance to chemotherapy drugs such as adriamycin[15], paclitaxel[16], cisplatin[17], tamoxifen[18] and trastuzumab[19] in breast cancer. The PTEN gene has multiple SNPs and is a highly polymorphic gene, and pathogenic germline mutations are found in all 9 exons[20]. In recent years there are several reports that polymorphism can affect the process of alternative splicing, which lead to the occurrence and development of many human diseases[21-23]. The process of AS is affected by many factors, among which the influence of the 3 'and 5 'splice site sequence of the branch point is common[24]. Studies have found that the PTEN gene can produce new isoforms through different splicing methods[25], but the reason for this abnormal alternative splicing is still unclear. It is currently believed that the splicing aberrations of the PTEN gene might be caused by the polymorphism of the standard splicing site.

Although many studies have shown the association between genetic polymorphisms of PTEN and drug resistance[26, 27], the underlying molecular mechanism of PTEN gene polymorphism leading to breast cancer chemotherapy resistance is still unclear. As far as we know, mutations in specific sites of genes can cause certain diseases and affect the AS process of genes, but the association between SNP and alternative splicing in Chinese population with the risk of breast cancer chemotherapy resistance has not been reported. Therefore, this study aims to explore the potential relationship between PTEN gene polymorphism and breast chemotherapy resistance, and investigate whether the SNP of PTEN gene affects alternative splicing of the gene. Our work might provide theoretical guidance for individualized treatment of breast cancer chemotherapy in Chinese population.

\section{Materials And Methods}

\subsection{Study population}

This study enrolled 234 samples, all from the former Lanzhou General Hospital of Lanzhou Military Region between September 2013 and April 2017. All samples were clinically diagnosed and received chemotherapy. All chemotherapy regimens were based on anthracyclines, samples who had not received treatment, did not use anthracyclines, or treated less than 2 courses were excluded. Usually anthracyclinebased chemotherapy regimens consist of 5-fluorouracil (5-FU), anthracycline compounds and cyclophosphamide (FAC-FEC-EC or AC regimen). After treatment, the chemotherapy efficacy after anthracycline treatment was evaluated and scored according to the Response Evaluation Criteria in Solid Tumor (RECIST) criteria[28]. In this study, sensitive patients were considered as patients with complete or partial remission, and patients with stable or progressing disease were classified as resistant patients.

The ethics committee of Lanzhou University School of Basic Medicine approved the study.

\subsection{Extraction of genomic DNA}


Collected peripheral blood of all samples in sterile tubes with EDTA and stored at $-80^{\circ} \mathrm{C}$. Used the phenol/chloroform method to isolate genomic DNA from white blood cells. The extracted DNA was stored at $-80^{\circ} \mathrm{C}$ until use. The concentration and purity of DNA were measured using NanoDrop 2000 C spectrophotometer (Thermo Fisher Scientific, Lenexa, KS, USA). Samples with a DNA concentration of more than 20ng/ $\mu$ l were used for genotyping (Beijing Bomiao Biotechnology Co., Ltd., China).

\subsection{Genotyping of PTEN polymorphism}

The DNA sample was handed over to the biological company to use Sequenom MassArray typing technology to performed genotyping of rs786204926. Used Assay Design 3.1 software to design the primers needed for Sequenom MassArray. Specific primer sequences: 1st -PCR primer: 5'-

ACGTTGGATGCTCTGGAATCCAGTGTTTCT-3', 2nd -PCR primer: 5'-

ACGTTGGATGTCTAGCTGTGGTGGGTTATG-3', UEP Sequences: 5'-ATGTTCTTCAAAAGGATATTGTGCAAC-

$3^{\prime}$. After PCR amplification reaction, SAP-PCR reaction, purification reaction, and spotting the purified extension product, Spectro CHIP was prepared. After spotting, used MALDI-TOF mass spectrometer to detect Spectro CHIP chip, and used TYPER4.0 software to analyze the results.

\subsection{Sequencing verification}

Randomly selected $10 \%$ of samples for verification. Designed primers and send them to company (General Biosystems (Anhui) Co., Ltd.) for synthesis. Primers were as follows: F: 5'GGGGAAAATAATACCTGGCTTCC-3', R: 5'-AGTTCGTCCCTTTCCAGCTT-3'. The PCR reaction program consists of 31 cycles, $94^{\circ} \mathrm{C} 3 \mathrm{~min} ; 94^{\circ} \mathrm{C} 30 \mathrm{~s} ; 59.6^{\circ} \mathrm{C} 30 \mathrm{~s} ; 72^{\circ} \mathrm{C} 25 \mathrm{~s} ; 72^{\circ} \mathrm{C} 5 \mathrm{~min}$. The synthesized products were electrophoresed on a $1 \%$ agarose gel, as shown in Table 7. Sequence verification of amplified PCR products and analysis of sequencing results (General Biosystems (Anhui) Co., Ltd.).

\subsection{In silico analysis}

In order to study the correlation between PTEN mutant genome and epirubicin drug sensitivity in breast cancer, we used Genomics of Drug Sensitivity in Cancer (GDSC) version 8.3 (https://www.cancerrxgene.org/)[29]. In order to predict whether rs786204926 affects the splicing site and splicing position changes, we used Alamut Visual v.2.15, Human Splicing Finder (HSF) (https://www.genomnis.com/access-hsf) and Berkeley Drosophila Genome Project (BDGP) (https://www.fruitfly.org/seq_tools/splice.html) conducted a computer analysis. CDNA and protein sequence refer to Ensembl Release 102, NCBI database. To translate cDNA sequence into amino acid sequence, we used online tool: https://www.novopro.cn/tools/translate.html. In order to predict the changes in the protein structure caused by the SNP at the splice site, we used SWISS-MODEL (https://swissmodel.expasy.org/interactive) for homology modelling[30]. In order to predict the change of protein flexibility after mutation, we used the online tool PredyFlexy (https://www.dsimb.inserm.fr/dsimb_tools/predyflexy/)

\subsection{Statistical analysis}


The Hardy-Weinberg balance test was performed on the allele frequency of rs786204926, and the $\chi 2$ test was used to determine whether the difference was statistically significant, $P<0.01$ was considered statistically significant. The clinical data and genotyping results were analysed using SPSS22.0 software (IBM Corp. Armonk, NY, USA), using independent sample t-test, chi-square test, and Fisher's exact test (sample size $<5$ ), $P<0.05$ was considered statistically significant.

\section{Results}

\subsection{Distributions of the clinical characteristics in breast cancer patients}

The study included 234 breast cancer patients, of which 157 were in the sensitive group (67.1\%) and 77 were in the resistance group (32.9\%). Table 1 showed the clinical characteristics and statistical information of 234 patients. All participants were female, and the median age was 48 years. According to AJCC Clinical Stage, most patients were diagnosed with stage II or stage III breast cancer. Pathological examination revealed 222 cases of invasive ductal carcinoma (94.9\%). There were statistical differences in Histological type, PR, and Molecular Subtype between the sensitive group and the resistant group ( $p<$ $0.05)$, and no significant difference was found in the other variables between the two groups $(p>0.05)$. 
Table 1

Characteristics of study subjects.

\begin{tabular}{|c|c|c|c|c|}
\hline Characteristics & Total & Sensitive & Resistance & $p$ \\
\hline & 234 & $157(0.671)$ & $77(0.329)$ & \\
\hline Age (years) & & & & 0.244 \\
\hline$<48$ & $113(0.483)$ & $80(0.510)$ & $33(0.429)$ & \\
\hline$\geq 48$ & $121(0.517)$ & $77(0.490)$ & $44(0.571)$ & \\
\hline Menopausal state & & & & 0.421 \\
\hline Menopause & $68(0.291)$ & $43(0.274)$ & $25(0.325)$ & \\
\hline Premenopausal & $166(0.709)$ & $114(0.726)$ & $52(0.675)$ & \\
\hline AJCC Clinical Stage & & & & 0.395 \\
\hline II & $104(0.444)$ & $64(0.408)$ & $40(0.519)$ & \\
\hline IIIA & $51(0.218)$ & $38(0.242)$ & $13(0.169)$ & \\
\hline IIIB & $65(0.278)$ & $45(0.287)$ & $20(0.260)$ & \\
\hline $\mathrm{IIIC}+\mathrm{IV}$ & $14(0.060)$ & $10(0.064)$ & $4(0.052)$ & \\
\hline Histological type & & & & $0.011 *$ \\
\hline Invasive ductal carcinoma & $222(0.949)$ & $153(0.975)$ & $69(0.896)$ & \\
\hline Other types & $12(0.051)$ & $4(0.025)$ & $8(0.104)$ & \\
\hline Tumor size & & & & 0.455 \\
\hline $\mathrm{T} 1($ size $\leq 2)$ & $64(0.274)$ & $39(0.248)$ & $25(0.325)$ & \\
\hline T2 (2\size $\leq 5)$ & $94(0.402)$ & $66(0.420)$ & $28(0.364)$ & \\
\hline T3(size $₫ 5)$ & $76(0.325)$ & $52(0.331)$ & $24(0.312)$ & \\
\hline Lymph node staging & & & & 0.981 \\
\hline NO & 79 (0.338) & $53(0.338)$ & $26(0.338)$ & \\
\hline N1 & $81(0.346)$ & $55(0.350)$ & $26(0.338)$ & \\
\hline N2 & $58(0.248)$ & $39(0.248)$ & $19(0.247)$ & \\
\hline N3 & $16(0.068)$ & $10(0.064)$ & $6(0.078)$ & \\
\hline
\end{tabular}

a C-erbB-2-positive: HER2 (+++) or Fish (+); ER, estrogen receptor; PR, progesterone receptor; C-erbB-2, human epidermal growth factor receptor 2 .

*: $P$ value $<0.05$ was considered as significant. 


\begin{tabular}{|c|c|c|c|c|}
\hline Characteristics & Total & Sensitive & Resistance & $p$ \\
\hline ER & & & & 0.181 \\
\hline Negative & $124(0.530)$ & $88(0.561)$ & $36(0.468)$ & \\
\hline Positive & $110(0.470)$ & $69(0.439)$ & $41(0.532)$ & \\
\hline PR & & & & $0.015^{*}$ \\
\hline Negative & $70(0.299)$ & $55(0.350)$ & $15(0.195)$ & \\
\hline Positive & $164(0.701)$ & $102(0.650)$ & $62(0.805)$ & \\
\hline C-erbB-2 ${ }^{a}$ & & & & 0.830 \\
\hline Negative & $32(0.137)$ & $22(0.140)$ & $10(0.130)$ & \\
\hline Positive & $202(0.863)$ & $135(0.860)$ & $67(0.870)$ & \\
\hline Molecular Subtype & & & & $0.041^{*}$ \\
\hline Luminal A & $50(0.214)$ & $29(0.185)$ & $21(0.273)$ & \\
\hline Luminal B & $131(0.560)$ & $98(0.624)$ & $33(0.429)$ & \\
\hline HER2 overexpression & $19(0.081)$ & $10(0.064)$ & $9(0.117)$ & \\
\hline Triple-negative & $34(0.145)$ & $20(0.127)$ & $14(0.182)$ & \\
\hline \multicolumn{5}{|c|}{$\begin{array}{l}\text { a C-erbB-2-positive: HER2 (+++) or Fish (+); ER, estrogen receptor; PR, progesterone receptor; C-erbB-2, } \\
\text { human epidermal growth factor receptor } 2 \text {. }\end{array}$} \\
\hline *: $P$ value $<0.05$ was & ignificant. & & & \\
\hline
\end{tabular}

\subsection{HW equilibrium test of rs786204926}

MassArray genotyping was performed on the rs786204926 polymorphism of 234 patients. We analyzed the genotype frequency of SNP, rs786204926 accords with the HW equilibrium state (Fig. 1B).

\subsection{Statistical analysis of rs786204926 polymorphism genotyping results}

Sequenom MassArray genotyping technology showed the genotypes of 234 participants, we further statistically analysed the genotype results and compared the distribution of genotypes under different genetic models. The results were listed in Table 2 . The number and frequency of the $A A, A G$, and $G G$ genotypes of the rs786204926 in the sensitive group were 85 (0.541), $56(0.357), 16(0.102)$; in the drugresistant group were 55 (0.714), 18 (0.234), 4 (0.052). In the heterozygote model, the AG genotype was significantly different from the AA genotype $(\mathrm{OR}=0.497,95 \% \mathrm{Cl}=0.265-0.933, P=0.002)$. Under the Dominant model, the $A G+G G$ genotype was significantly different from the AA genotype $(O R=0.472$, 
$95 \% \mathrm{Cl}=0.263-0.848, P=0.011)$. In the allele analysis, there was a statistical difference between alleles $\mathrm{G}$ and $\mathrm{A}(\mathrm{OR}=0.522,95 \% \mathrm{Cl}=0.320-0.850, P=0.008)$, Carrying the $\mathrm{G}$ allele or $\mathrm{AG}$ genotype will reduce the risk of breast cancer resistance based on anthracycline chemotherapy.

Table 2

The association between PTEN gene polymorphisms and the risk of breast cancer chemotherapy resistance.

\begin{tabular}{|c|c|c|c|c|c|}
\hline Model & Allele/Genotype & Sensitive & Resistive & OR $(95 \% \mathrm{Cl})$ & $P$ \\
\hline \multirow[t]{2}{*}{ Heterozygote } & AA & $85(0.541)$ & $55(0.714)$ & & \\
\hline & $A G$ & $56(0.357)$ & $18(0.234)$ & $0.497(0.265-0.933)$ & 0.028 \\
\hline \multirow[t]{2}{*}{ Homozygote } & AA & $85(0.541)$ & $55(0.714)$ & & \\
\hline & GG & $16(0.102)$ & $4(0.052)$ & $0.386(0.123-1.216)$ & $0.136^{*}$ \\
\hline \multirow[t]{2}{*}{ Dominant } & AA & $85(0.541)$ & $55(0.714)$ & & \\
\hline & $A G+G G$ & $72(0.459)$ & $22(0.286)$ & $0.472(0.263-0.848)$ & 0.011 \\
\hline \multirow[t]{2}{*}{ Recessive } & GG & $16(0.102)$ & $4(0.052)$ & & \\
\hline & $A G+A A$ & $141(0.898)$ & $73(0.948)$ & $2.071(0.668-6.421)$ & $0.225^{\star}$ \\
\hline \multirow[t]{2}{*}{ Additive } & $A G$ & $56(0.357)$ & $18(0.234)$ & & \\
\hline & $\mathrm{GG}+\mathrm{AA}$ & $101(0.643)$ & $59(0.766)$ & $1.817(0.977-3.380)$ & 0.057 \\
\hline \multirow[t]{2}{*}{ Allele } & A & $226(0.720)$ & $128(0.831)$ & & \\
\hline & G & $88(0.280)$ & $26(0.169)$ & $0.522(0.320-0.850)$ & 0.008 \\
\hline \multicolumn{6}{|c|}{ OR, Odds ratio; $\mathrm{Cl}$, Confidence interval. } \\
\hline \multicolumn{6}{|c|}{ *: Pass Fisher's exact test calculation; } \\
\hline \multicolumn{6}{|c|}{$P$ value $<0.05$ was considered as significant. } \\
\hline
\end{tabular}

\subsection{Sanger sequence verification}

To verify the results of the genotyping method, we randomly selected some samples to use Sanger sequencing method to verify. Figure $1 \mathrm{~A}$ showed the image of PCR products of some samples after agarose gel electrophoresis. The wild homozygous, heterozygous, and mutant homozygous sequence of the rs786204926 polymorphism were shown in Fig. 1C. 


\subsection{Analysis mutation of the PTEN gene and its association with drug sensitivity}

According to The cBioPortal for Cancer Genomics (http://cbioportal.org) database analysis of samples with mutation data in invasive breast cancer (TCGA, Firehose Legacy) [31, 32], PTEN had mutations in 9\% of patients, The database had reported that there are two splice sites (X70_splice, X212_splice) on the PTEN gene. We predicted that the rs786204926 is also a splice site (Fig. 2). In order to study the correlation between the PTEN mutant genome and epirubicin drug sensitivity in breast cancer, we used GDSC online software for analysis (Supplementary Table 1). The results showed that PTEN mutant genome would increase the sensitivity of epirubicin drug in breast cancer (Fig. 3).

\section{6 rs786204926 polymorphism affected AS of PTEN gene}

Mutations at the splicing site might affect alternative splicing of genes. These effects had been detected by Alamut Visual v.2.15, HSF and BDGP. HSF predicted whether the rs786204926A $>$ G mutation affected AS. The prediction results showed that rs786204926 is a splice acceptor site, when carrying G allele, it will destroy the wild-type acceptor site and affect splicing (Supplementary Table 2). BDGP was used to predict the splice site changes of SNPs (0.4 as the threshold). The results showed that the donor site remains unchanged after the SNP mutation. CAAAAAG-GT-AAGTTA was the main donor site (score = 1.00); The acceptor site generated a new splice site after SNP mutation (GTTTTTTTTTCTTATTCTG-AGGTTATCTTTTTACCACGGTT), the wild splice site disappeared (GAGGTTATCTTTTTACCAC-AGTTGCACAATATCCTTTTGAA) (Supplementary Table 3). Further analysis using Alamut Visual v.2.14 showed that the rs786204926A > G mutation destroyed the acceptor site and created a new acceptor site. It also showed the changes between the exon splicing enhancers (ESE) after mutation (Fig. 4A), the results showed that the ESE binding ability is weakened after mutation. According to the changes of the splicing site after mutation, we speculated the amino acid sequence after mutation (Fig. 4B).

\subsection{Predict that SNP mutations cause structural changes in PTEN protein}

According to the predicted amino acid sequence, we used SWISS-MODEL for homology modeling and predicted the changes in the protein structure after mutation (Fig. 5). The results showed the spatial structure of the protein was changed after gene mutation. Then we used PredyFlexy to analyze the protein flexibility of the rs786204926A $>\mathrm{G}$ mutant protein sequence and compared it with the original sequence. After the mutation, the flexibility of the PTEN protein had a certain change (Fig. 6). In the structure of PTEN protein, the N-terminal phosphatase domain is closely related to its phosphatase function. This domain is composed of amino acids 1-185 of the $\mathrm{N}$-terminal, and amino acids 123-130 constitute the core of its phosphatase. Among the core amino acids, arginine changes from flexible to intermediate, which will affect the function of phosphatase. 


\section{Discussion}

PTEN is the first reported tumor suppressor gene with phosphatase activity, which plays a vital role in the cell apoptosis, cell cycle arrest and cell migration.[33,34]. Therefore, PTEN gene plays an important role in the mechanism of multi-drug resistance in cancers[35]. Previous studies have shown that PTEN rs701848 polymorphism might be a candidate pharmacogenomic factor to assess the susceptibility of breast cancer and response and prognosis prediction for chemotherapy in breast cancer [36]. Therefore, it was shown that PTEN gene polymorphism was related to chemotherapy resistance of breast cancer. In recent years, it had been found that PTEN gene polymorphism and chemotherapy resistance are different in breast cancer. However, the potential association between rs786204926 and chemotherapy resistance in breast cancer has not been reported. In our research, we selected PTEN gene polymorphism rs786204926 to study the potential association between PTEN gene polymorphism and chemotherapy resistance in breast cancer. Our research showed that rs786204926 polymorphism was closely related to breast cancer chemotherapy resistance, and found that the AG Heterozygote rs786204926 mutation has decreased risk of breast cancer resistance to anthracycline-based chemotherapy drugs compared with AA/GG individuals. $G$ allele in patients with chemotherapy reduces the risk of drug resistance. Previous studies have confirmed that the association between genetic polymorphisms and breast cancer varies greatly among different races, indicating that different genetic backgrounds have different mutation frequencies[37]. In addition, studies have shown that genetic SNP might change the response to drugs, and there are significant differences between different races[38]. This indicates that mutations have different therapeutic effects on people with different genetic backgrounds. China has a vast territory and a large population. Different regions have different eating habits, cultural backgrounds, living environments, and genetic backgrounds. Our samples mainly collected patients from Northwest China. This study is the first time to detect and analyse the PTEN gene polymorphism of rs786204926 in Northwest China, and provide important theoretical data for establishing a database of PTEN gene polymorphism in Northwest China and promoting pharmacogenomics research to achieve personalized medicine.

Compared with previous studies related to chemotherapy resistance, our research not only analysed gene polymorphism, but also used software to predict the correlation between rs786204926 and AS. Up to 50\% of all mutations leading to genetic diseases result in abnormal splicing[39]. It has been found that PTEN splicing aberrations might be caused by genetic mutations at the junction of the splicing sites and deep in the introns[40]. The function of splice sites is to "signal" the assembling snRNAs and auxiliary splicing factors to recognize the "staging area" in order to initiate the assembly of the spliceosome eventually leading to the excision of intron and joining of exons to yield a mature mRNA[41]. This is one of the important mechanisms of alternative splicing involved in the occurrence and development of various diseases. Preliminary studies showed that the impact of genetic polymorphism mutations had been analysed through silicon prediction tools[42, 43]. Therefore, we used silicon analysis to predict the rs786204926 polymorphism to generate new splice sites. The bioinformatics software predicted that rs786204926 is a potential splice acceptor site. When an A>G mutation occurs at this site, it will destroy the wild-type acceptor site to create a new acceptor site and affect alternative splicing. After mutation, 
there are 18 bases from Pre-mRNA intron 4 will be retained on the mature mRNA, and the exon splicing enhancer (ESE) binding ability will also change, which might eventually cause the mutant protein to have 6 amino acids more than the wild type. In the structure of PTEN protein, the N-terminal phosphatase domain composed of $\mathrm{N}$-terminal amino acids 1-185 is closely related to its phosphatase function[44]. The 6 amino acids produced by the mutant are exactly in this domain, which will cause the protein structure to change. Amino acids 123-130 (HCxxGxxR) are the core sequence of its phosphatase[34]. After mutation, we found that there are varying degrees of protein flexibility changes in these amino acids, especially the decrease of protein flexibility in the core sequence. Flexibility is necessary for a protein to perform its biological function[ $[45,46]$. If the flexibility is reduced, the conformational state of the protein will change greatly, leading to the reduction or even loss of its phosphatase function. PTEN is a tumor suppressor gene with phosphatase activity[34]. On the cell membrane, PTEN mediates the conversion of 3,4,5-phosphatidylinositol triphosphate (PIP3) to PIP2, and inhibits the PI3K/Akt pathway to arrest the cell cycle In the $\mathrm{G} 1$ phase[47-49]. When rs786204926A > G is mutated, it might cause changes in mRNA and protein through alternative splicing, and ultimately lead to the reduction or loss of PTEN phosphatase function. Loss of PTEN function will lose its negative regulatory effect on PI3K/Akt, Akt will be continuously activated, the cell cycle will be accelerated, and cell growth and proliferation will be accelerated, reducing the risk of breast cancer chemotherapy resistance. Therefore, we suspect that the chemotherapy resistance of breast cancer patients is mainly caused by the AS of the PTEN gene rs786204926 polymorphism.

Previous studies mainly collected clinical information and blood samples, test results, and statistical analysis to obtain the association between gene polymorphism and breast cancer chemotherapy resistance. However, the mechanism of chemotherapy resistance has not been further studied. In the current research, we have not only conducted association studies, but also predicted the function of SNPs in chemotherapy resistance-related genes by silico analysis. It is predicted that rs 786204926 might affect the alternative splicing of PTEN gene (Fig. 5). Although this study has conducted in-depth studies on the potential molecular mechanisms of PTEN gene polymorphism and breast cancer chemotherapy resistance, its limitations should be recognized. The above prediction results require specific experiments to support our findings.

\section{Conclusions}

In conclusion, our study hypothesize that the mechanism of breast cancer chemotherapy resistance might be caused by a change in the alternative splicing caused by the rs786204926 of the PTEN gene. Our work not only provides theoretical guidance for the individualized treatment of clinical breast cancer patients in the Chinese population, but also provides new ideas for the mechanism of breast cancer chemotherapy resistance.

\section{Abbreviations}


SNP: Single nucleotide polymorphism; AS: Alternative splicing; PCR: Polymerase chain reaction; ESE: Exon splicing enhancer; Cl: Confidence intervals; OR: Odds ratio; HW: Hardy-Weinberg; AJCC: American Joint Committee on Cancer; ER: Estrogen receptor; PR: Progesterone receptor; C-erbB-2: human epidermal growth factor receptor 2 .

\section{Declarations}

\section{Acknowledgements}

Gansu Province Science and Technology Innovation Service Platform Construction Project (18JR2TA024), the national Key R\&D project of the Chinese

Ministry of Science and Technology (2018YFE0205100), the Key Program of the National Natural Science Foundation of China (U1632270), the National Natural Science Foundation of China (11675234), and the Science and Technology Plan Project of Lanzhou (2019-4-7).

\section{Competing interests}

The authors declare that they have no conflict of interest.

\section{References}

1. Siegel RL, Miller KD, Jemal A. Cancer statistics, 2018. CA: a cancer journal for clinicians. 2018;68(1):7-30.

2. Carioli G, Malvezzi M, Rodriguez T, Bertuccio P, Negri E, La Vecchia C. Trends and predictions to 2020 in breast cancer mortality: Americas and Australasia. Breast (Edinburgh, Scotland). 2018;37:163-9.

3. Chen W, Zheng R, Baade PD, Zhang S, Zeng H, Bray F, et al. Cancer statistics in China, 2015. CA: a cancer journal for clinicians. 2016;66(2):115-32.

4. Holohan C, Van Schaeybroeck S, Longley DB, Johnston PG. Cancer drug resistance: an evolving paradigm. Nature reviews Cancer. 2013;13(10):714-26.

5. Crystal AS, Shaw AT, Sequist LV, Friboulet L, Niederst MJ, Lockerman EL, et al. Patient-derived models of acquired resistance can identify effective drug combinations for cancer. Science (New York, NY). 2014;346(6216):1480-6.

6. Li M, Wu XM, Gao J, Yang F, Zhang CL, Ke K, et al. Mutations in the P10 region of procaspase-8 lead to chemotherapy resistance in acute myeloid leukemia by impairing procaspase- 8 dimerization. Cell death \& disease. 2018;9(5):516.

7. Rosell R, Felip E. Predicting response to paclitaxel/carboplatin-based therapy in non-small cell lung cancer. Seminars in oncology. 2001;28(4 Suppl 14):37-44.

8. Steelman LS, Navolanic PM, Sokolosky ML, Taylor JR, Lehmann BD, Chappell WH, et al. Suppression of PTEN function increases breast cancer chemotherapeutic drug resistance while conferring sensitivity to mTOR inhibitors. Oncogene. 2008;27(29):4086-95. 
9. Liu Y, Yang EJ, Zhang B, Miao Z, Wu C, Lyu J, et al. PTEN deficiency confers colorectal cancer cell resistance to dual inhibitors of FLT3 and aurora kinase A. Cancer letters. 2018;436:28-37.

10. Cheng F, Eng C. PTEN Mutations Trigger Resistance to Immunotherapy. Trends in molecular medicine. 2019;25(6):461-3.

11. Fletcher JI, Williams RT, Henderson MJ, Norris MD, Haber M. ABC transporters as mediators of drug resistance and contributors to cancer cell biology. Drug resistance updates : reviews and commentaries in antimicrobial and anticancer chemotherapy. 2016;26:1-9.

12. Pajic M, lyer JK, Kersbergen A, van der Burg E, Nygren AO, Jonkers J, et al. Moderate increase in Mdr1a/1b expression causes in vivo resistance to doxorubicin in a mouse model for hereditary breast cancer. Cancer research. 2009;69(16):6396-404.

13. Wein L, Loi S. Mechanisms of resistance of chemotherapy in early-stage triple negative breast cancer (TNBC). Breast (Edinburgh, Scotland). 2017;34 Suppl 1:S27-s30.

14. Hollander MC, Blumenthal GM, Dennis PA. PTEN loss in the continuum of common cancers, rare syndromes and mouse models. Nature reviews Cancer. 2011;11(4):289-301.

15. Wang ZX, Lu BB, Wang H, Cheng ZX, Yin YM. MicroRNA-21 modulates chemosensitivity of breast cancer cells to doxorubicin by targeting PTEN. Archives of medical research. 2011;42(4):281-90.

16. Liu L, Meng T, Zheng X, Liu Y, Hao R, Yan Y, et al. Transgelin 2 Promotes Paclitaxel Resistance, Migration, and Invasion of Breast Cancer by Directly Interacting with PTEN and Activating PI3K/Akt/GSK-3 $\beta$ Pathway. Molecular cancer therapeutics. 2019;18(12):2457-68.

17. Gao C, Yuan X, Jiang Z, Gan D, Ding L, Sun Y, et al. Regulation of AKT phosphorylation by GSK3 $\beta$ and PTEN to control chemoresistance in breast cancer. Breast cancer research and treatment. 2019;176(2):291-301.

18. Gao Y, Li X, Zeng C, Liu C, Hao Q, Li W, et al. CD63(+) Cancer-Associated Fibroblasts Confer Tamoxifen Resistance to Breast Cancer Cells through Exosomal miR-22. Advanced science (Weinheim, Baden-Wurttemberg, Germany). 2020;7(21):2002518.

19. Gong C, Yao Y, Wang Y, Liu B, Wu W, Chen J, et al. Up-regulation of miR-21 mediates resistance to trastuzumab therapy for breast cancer. The Journal of biological chemistry. 2011;286(21):19127-37.

20. Yehia L, Keel E, Eng C. The Clinical Spectrum of PTEN Mutations. Annual review of medicine. 2020;71:103-16.

21. Xiao F, Zhang P, Wang Y, Tian Y, James M, Huang CC, et al. Single-nucleotide polymorphism rs13426236 contributes to an increased prostate cancer risk via regulating MLPH splicing variant 4. Molecular carcinogenesis. 2020;59(1):45-55.

22. Fairbrother WG, Holste D, Burge CB, Sharp PA. Single nucleotide polymorphism-based validation of exonic splicing enhancers. PLoS biology. 2004;2(9):E268.

23. Knudsen KE, Diehl JA, Haiman CA, Knudsen ES. Cyclin D1: polymorphism, aberrant splicing and cancer risk. Oncogene. 2006;25(11):1620-8. 
24. Halim D, Brosens E, Muller F, Wangler MF, Beaudet AL, Lupski JR, et al. Loss-of-Function Variants in MYLK Cause Recessive Megacystis Microcolon Intestinal Hypoperistalsis Syndrome. American journal of human genetics. 2017;101(1):123-9.

25. Agrawal S, Eng C. Differential expression of novel naturally occurring splice variants of PTEN and their functional consequences in Cowden syndrome and sporadic breast cancer. Human molecular genetics. 2006;15(5):777-87.

26. Wang C, Yang D, Zhang X, Zhang X, Yang L, Wang P, et al. Association of PTEN Gene SNPs rs2299939 With PFS in Patients With Small Cell Lung Cancer Treated With Early Radiotherapy. Frontiers in genetics. 2020;11:298.

27. Wang F, Diao XY, Zhang X, Shao Q, Feng YF, An X, et al. Identification of genetic alterations associated with primary resistance to EGFR-TKIs in advanced non-small-cell lung cancer patients with EGFR sensitive mutations. Cancer communications (London, England). 2019;39(1):7.

28. Eisenhauer EA, Therasse P, Bogaerts J, Schwartz LH, Sargent D, Ford R, et al. New response evaluation criteria in solid tumours: revised RECIST guideline (version 1.1). European journal of cancer (Oxford, England : 1990). 2009;45(2):228-47.

29. Yang W, Soares J, Greninger P, Edelman EJ, Lightfoot H, Forbes S, et al. Genomics of Drug Sensitivity in Cancer (GDSC): a resource for therapeutic biomarker discovery in cancer cells. Nucleic acids research. 2013;41(Database issue):D955-61.

30. Waterhouse A, Bertoni M, Bienert S, Studer G, Tauriello G, Gumienny R, et al. SWISS-MODEL: homology modelling of protein structures and complexes. Nucleic acids research. 2018;46(W1):W296-w303.

31. Gao J, Aksoy BA, Dogrusoz U, Dresdner G, Gross B, Sumer So, et al. Integrative analysis of complex cancer genomics and clinical profiles using the cBioPortal. Science signaling. 2013;6(269):pl1.

32. Cerami E, Gao J, Dogrusoz U, Gross BE, Sumer SO, Aksoy BA, et al. The cBio cancer genomics portal: an open platform for exploring multidimensional cancer genomics data. Cancer discovery. 2012;2(5):401-4.

33. Cantley LC, Neel BG. New insights into tumor suppression: PTEN suppresses tumor formation by restraining the phosphoinositide 3-kinase/AKT pathway. Proceedings of the National Academy of Sciences of the United States of America. 1999;96(8):4240-5.

34. Worby CA, Dixon JE. PTEN. Annual review of biochemistry. 2014;83:641-69.

35. Wu H, Wang K, Liu W, Hao Q. PTEN overexpression improves cisplatin-resistance of human ovarian cancer cells through upregulating KRT10 expression. Biochemical and biophysical research communications. 2014;444(2):141-6.

36. Li X, Zhang R, Liu Z, Li S, Xu H. The genetic variants in the PTEN/PI3K/AKT pathway predict susceptibility and $\mathrm{CE}(\mathrm{A}) \mathrm{F}$ chemotherapy response to breast cancer and clinical outcomes. Oncotarget. 2017;8(12):20252-65.

37. Cai Q, Wen W, Qu S, Li G, Egan KM, Chen K, et al. Replication and functional genomic analyses of the breast cancer susceptibility locus at 6q25.1 generalize its importance in women of chinese, 
Japanese, and European ancestry. Cancer research. 2011;71(4):1344-55.

38. Sandanaraj E, Lal S, Selvarajan V, Ooi LL, Wong ZW, Wong NS, et al. PXR pharmacogenetics: association of haplotypes with hepatic CYP3A4 and ABCB1 messenger RNA expression and doxorubicin clearance in Asian breast cancer patients. Clinical cancer research : an official journal of the American Association for Cancer Research. 2008;14(21):7116-26.

39. Cartegni L, Chew SL, Krainer AR. Listening to silence and understanding nonsense: exonic mutations that affect splicing. Nature reviews Genetics. 2002;3(4):285-98.

40. Agrawal S, Pilarski R, Eng C. Different splicing defects lead to differential effects downstream of the lipid and protein phosphatase activities of PTEN. Human molecular genetics. 2005;14(16):2459-68.

41. Shukla GC, Singh J. Mutations of RNA splicing factors in hematological malignancies. Cancer letters. 2017;409:1-8.

42. Wappenschmidt B, Becker AA, Hauke J, Weber U, Engert S, Köhler J, et al. Analysis of 30 putative BRCA1 splicing mutations in hereditary breast and ovarian cancer families identifies exonic splice site mutations that escape in silico prediction. PloS one. 2012;7(12):e50800.

43. Zhang S, Wang J, Zhang A, Zhang X, You T, Xie D, et al. A SNP involved in alternative splicing of $A B C B 1$ is associated with clopidogrel resistance in coronary heart disease in Chinese population. Aging. 2020;12(24):25684-99.

44. Hopkins BD, Hodakoski C, Barrows D, Mense SM, Parsons RE. PTEN function: the long and the short of it. Trends in biochemical sciences. 2014;39(4):183-90.

45. de Brevern AG, Bornot A, Craveur P, Etchebest C, Gelly JC. PredyFlexy: flexibility and local structure prediction from sequence. Nucleic acids research. 2012;40(Web Server issue):W317-22.

46. Narwani TJ, Etchebest C, Craveur P, Léonard S, Rebehmed J, Srinivasan N, et al. In silico prediction of protein flexibility with local structure approach. Biochimie. 2019;165:150-5.

47. Carnero A, Paramio JM. The PTEN/PI3K/AKT Pathway in vivo, Cancer Mouse Models. Frontiers in oncology. 2014;4:252.

48. Furnari FB, Huang HJ, Cavenee WK. The phosphoinositol phosphatase activity of PTEN mediates a serum-sensitive G1 growth arrest in glioma cells. Cancer research. 1998;58(22):5002-8.

49. Jin $H$, Sun $Y$, Wang $S$, Cheng $X$. Matrine activates PTEN to induce growth inhibition and apoptosis in V600EBRAF harboring melanoma cells. International journal of molecular sciences.

2013;14(8):16040-57.

\section{Figures}




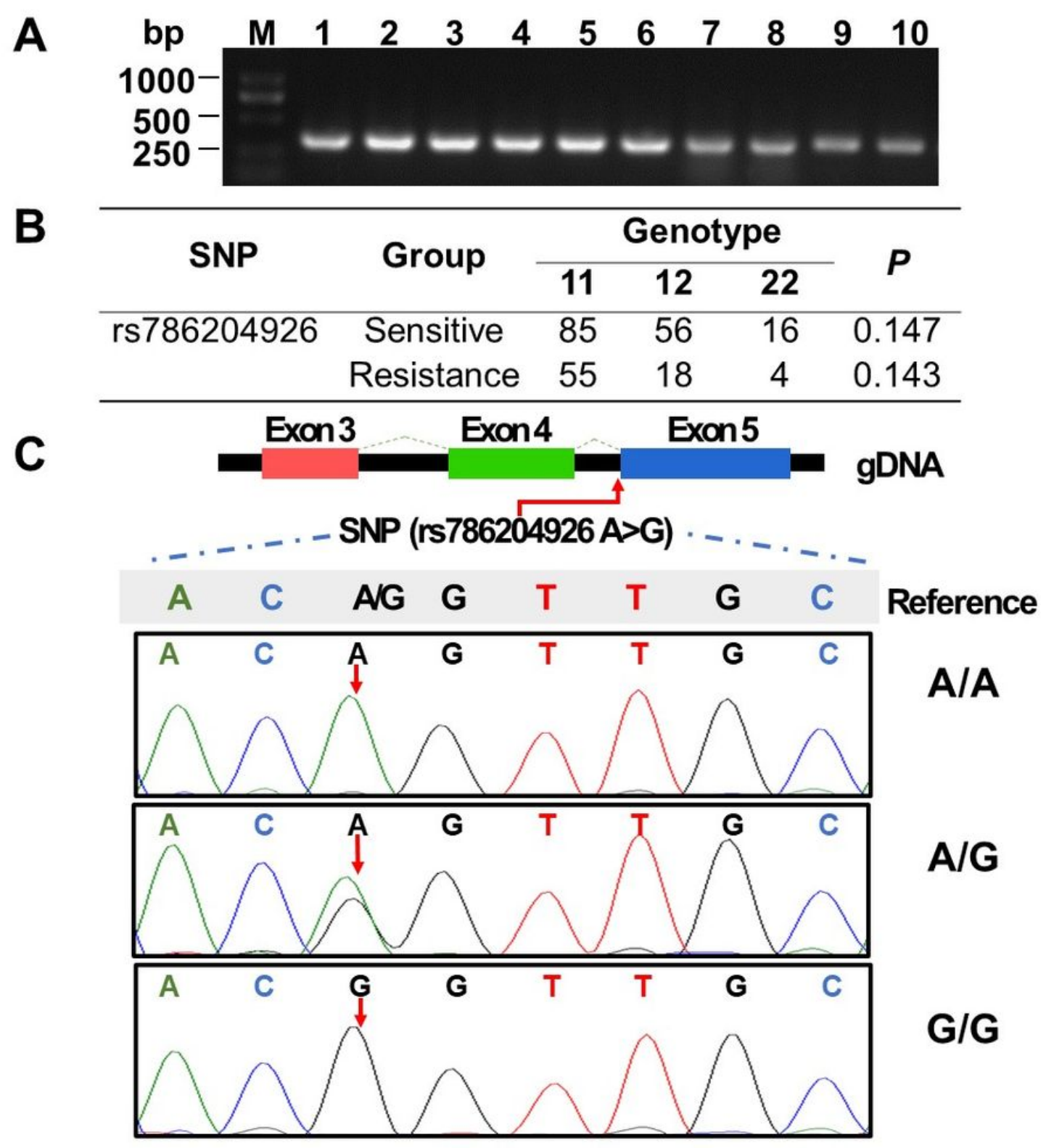

Figure 1

Electropherograms of PCR product fragments verified by sequence verification of SNPs of partial DNA samples. (A) Electropherogram of PCR product of rs786204926 polymorphism. (B) The test of HardyWeinberg Equilibrium of rs786204926. (C) Sequence verification of rs786204926 polymorphism: GG-wild type homozygous, AG-heterozygote, AA-SNP homozygous. The arrow in the picture points to the specific bases of the rs786204926 polymorphic sequence. 


\section{A PTEN 9\% ||||||||||||||||||||||||||||||||||||||||||||||||||||||||||-|, \\ Genetic Alteration \\ Inframe Mutation (unknown significance) \\ Missense Mutation (unknown significance) \\ Amplification \\ Deep Deletion \\ Missense Mutation (putative driver) \\ Truncating Mutation (putative driver) \\ No alterations}

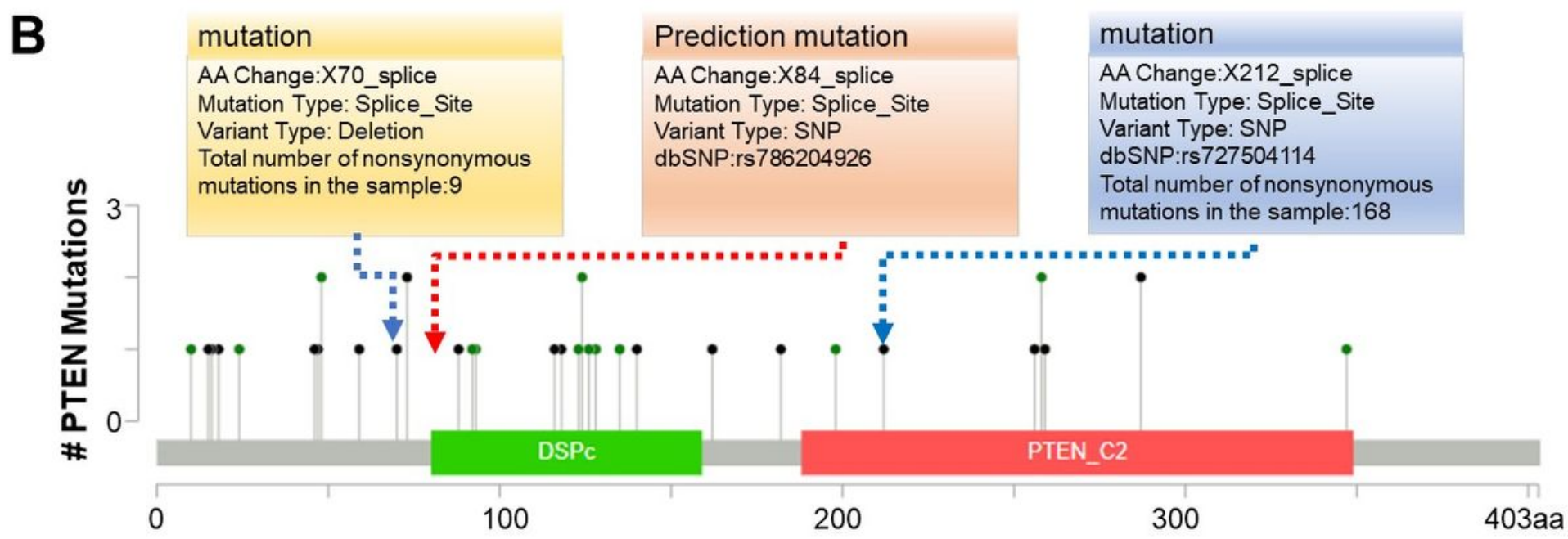

Figure 2

PTEN mutations in breast cancer. (A) Genetic alteration of PTEN in breast cancer from cBio Portal of Cancer Genome Atlas (TCGA). (B) The sites and frequencies of PTEN mutations in breast cancer were obtained from the cBio Portal of The Cancer Genome Atlas (TCGA). Information on mutations at the splice site are indicated in the box. 

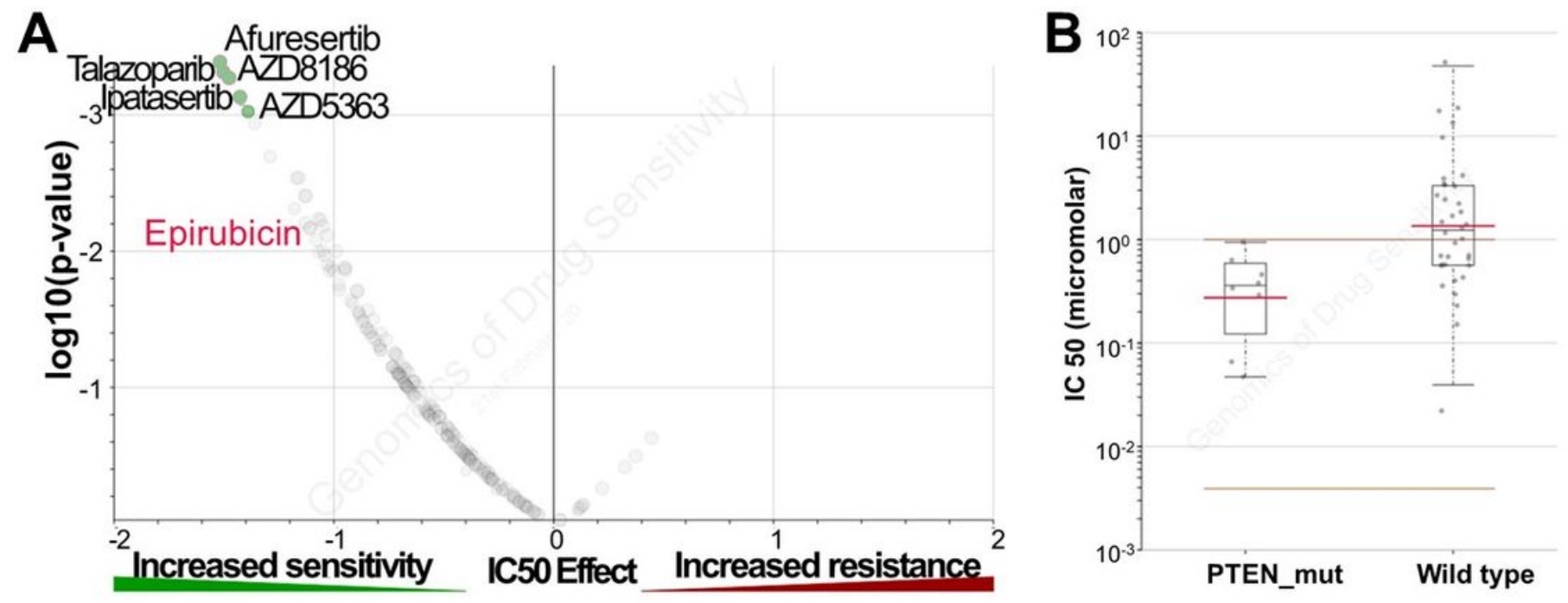

\begin{tabular}{|c|c|c|c|c|c|}
\hline Drug & Drug Target & $\begin{array}{c}\text { Effect } \\
\text { size }\end{array}$ & P-value & FDR\% & $\begin{array}{c}\text { No. of altered } \\
\text { cell lines }\end{array}$ \\
\hline Afuresertib & AKT1, AKT2, AKT3 & -1.49 & 0.000433 & 1.73 & 8 \\
\hline Talazoparib & PARP1, PARP2 & -1.48 & 0.000518 & 2.43 & 8 \\
\hline AZD8186 & PI3Kalpha, PI3Kbeta & -1.46 & 0.00057 & 0.57 & 8 \\
\hline Ipatasertib & AKT1, AKT, AKT3 & -1.42 & 0.000767 & 3.61 & 8 \\
\hline AZD5363 & AKT1, AKT2, AKT3, ROCK2 & -1.39 & 0.000945 & 4.44 & 8 \\
\hline Pyridostatin & G-quadruplex stabiliser & -1.36 & 0.00116 & 4.02 & 8 \\
\hline Uprosertib & AKT1, AKT2, AKT3 & -1.29 & 0.00203 & 6.1 & 8 \\
\hline Crizotinib & MET, ALK, ROS1 & -1.16 & 0.00289 & 4.95 & 9 \\
\hline NU7441 & DNAPK & -1.17 & 0.00291 & 4.65 & 9 \\
\hline Oxaliplatin & DNA alkylating agent & -1.13 & 0.0039 & 18.7 & 9 \\
\hline EPZO004777 & DOT1L & -1.13 & 0.00395 & 7.9 & 9 \\
\hline Entospletinib & SYK & -1.18 & 0.00485 & 9.69 & 8 \\
\hline Tozasertib & AURKA, AURKB, AURKC, others & -1.07 & 0.0058 & 4.51 & 9 \\
\hline Axitinib & PDGFR, KIT, VEGFR & -1.06 & 0.00593 & 30.8 & 9 \\
\hline GSK2578215A & LRRK2 & -1.13 & 0.00618 & 6.18 & 8 \\
\hline Cisplatin & DNA crosslinker & -1.05 & 0.00656 & 18.6 & 9 \\
\hline AT13148 & AKT1 & -1.11 & 0.00676 & 31.8 & 8 \\
\hline Epirubicin & Anthracycline & -1.11 & 0.00679 & 16 & 8 \\
\hline $\mathrm{RO}-3306$ & CDK1 & -1.03 & 0.00764 & 11.5 & 9 \\
\hline Rapamycin & MTORC1 & -1.08 & 0.00824 & 33 & 8 \\
\hline AMG-319 & PI3K (beta sparing) & -1.05 & 0.00989 & 19.8 & 8 \\
\hline
\end{tabular}

\section{Figure 3}

Genomics of Drug Sensitivity in Cancer (GDSC) was used to analyze the relationship between PTEN mutation genome and drug sensitivity in breast cancer. (A) The association of PTEN mutation genome and chemotherapeutic drug sensitivity in breast cancer. Each circle represents an association between a genomic marker and drug sensitivity analysed using ANOVA. (B) The relationship between the IC50 of epirubicin in breast cancer and the PTEN mutation genome. (C) Drugs related to PTEN mutant genome and breast cancer chemotherapy drug sensitivity. 

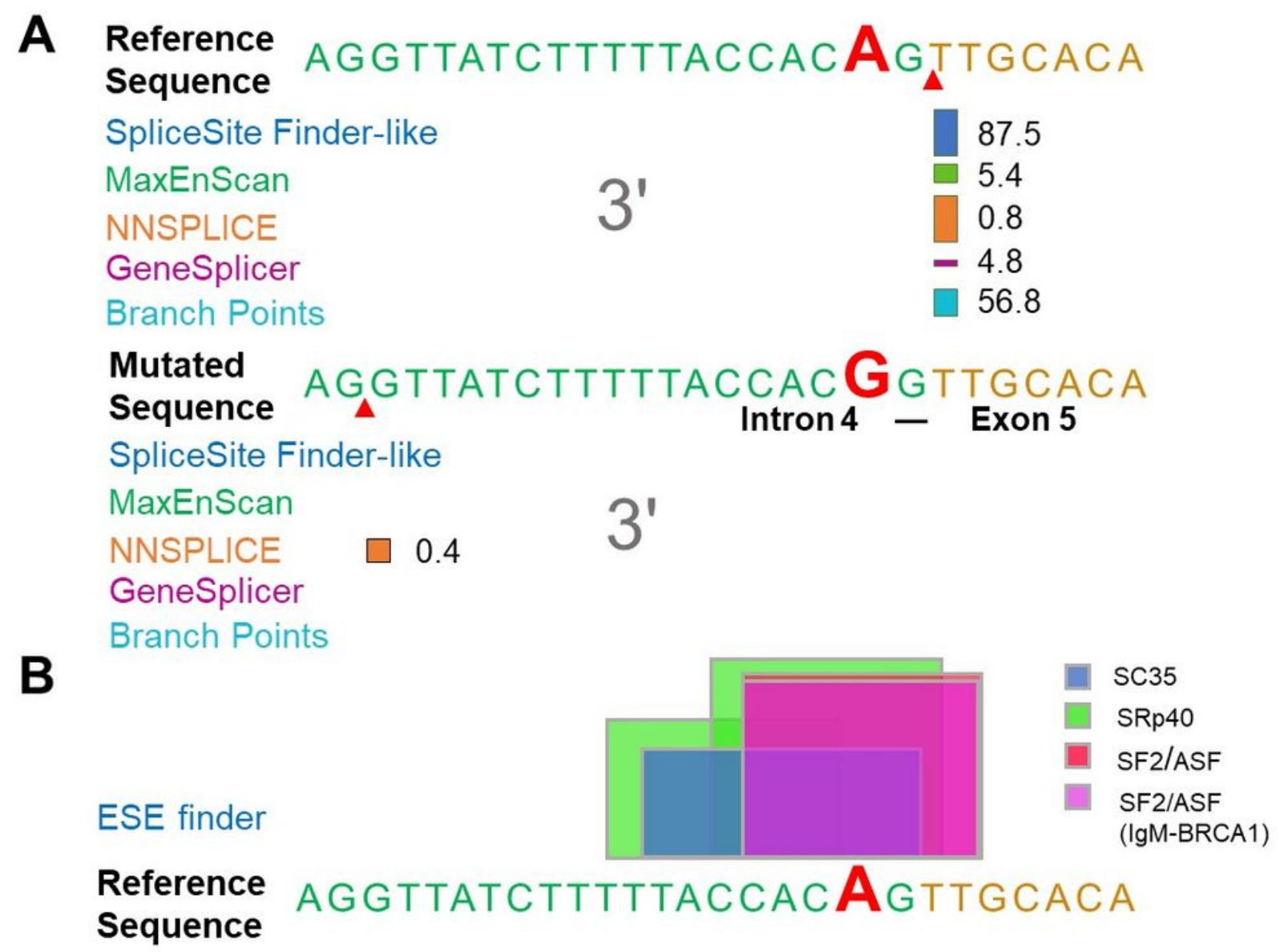

ESE finder

Mutated Sequence

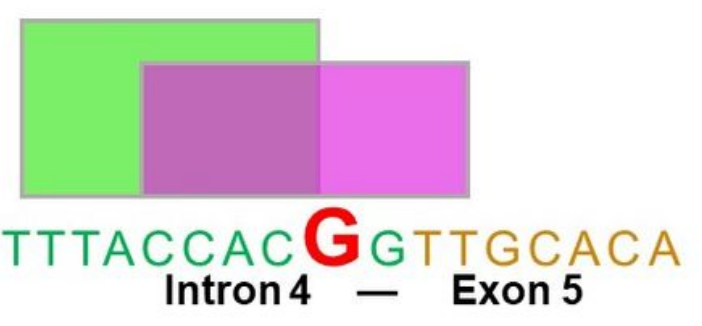

\section{Figure 4}

Using Alamut Visual to analyze the effect of rs786204926 on alternative splicing of the PTEN gene. (A) rs786204926A>G destroys the original 3' splice site and creates a new 3' splice site. The triangle represents the splice site. (B) rs786204926A>G mutation after the subtle difference between the splicing enhancer (ESE). 


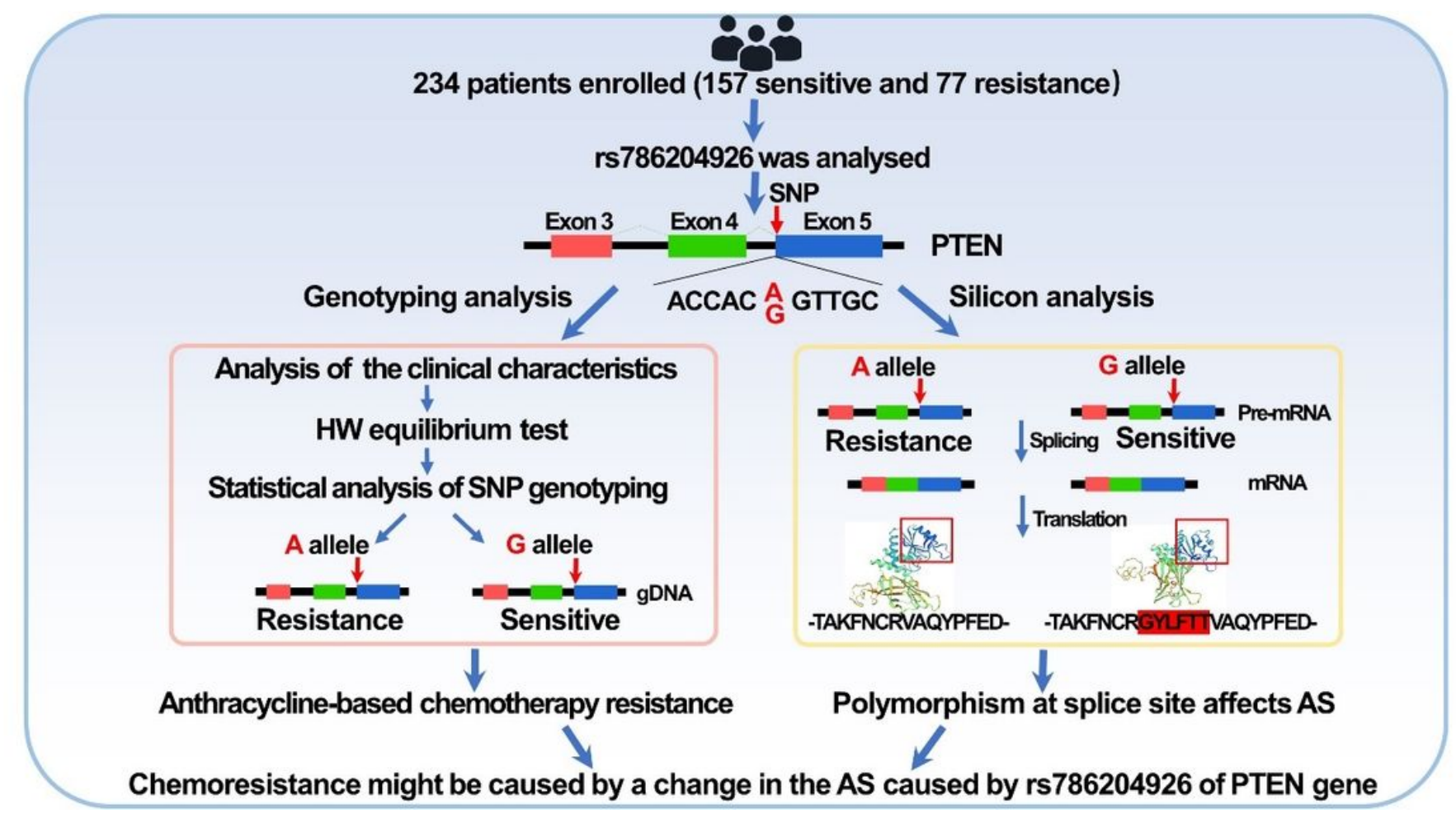

\section{Figure 5}

Chemoresistance might be caused by a change in the AS caused by rs786204926 of PTEN gene 

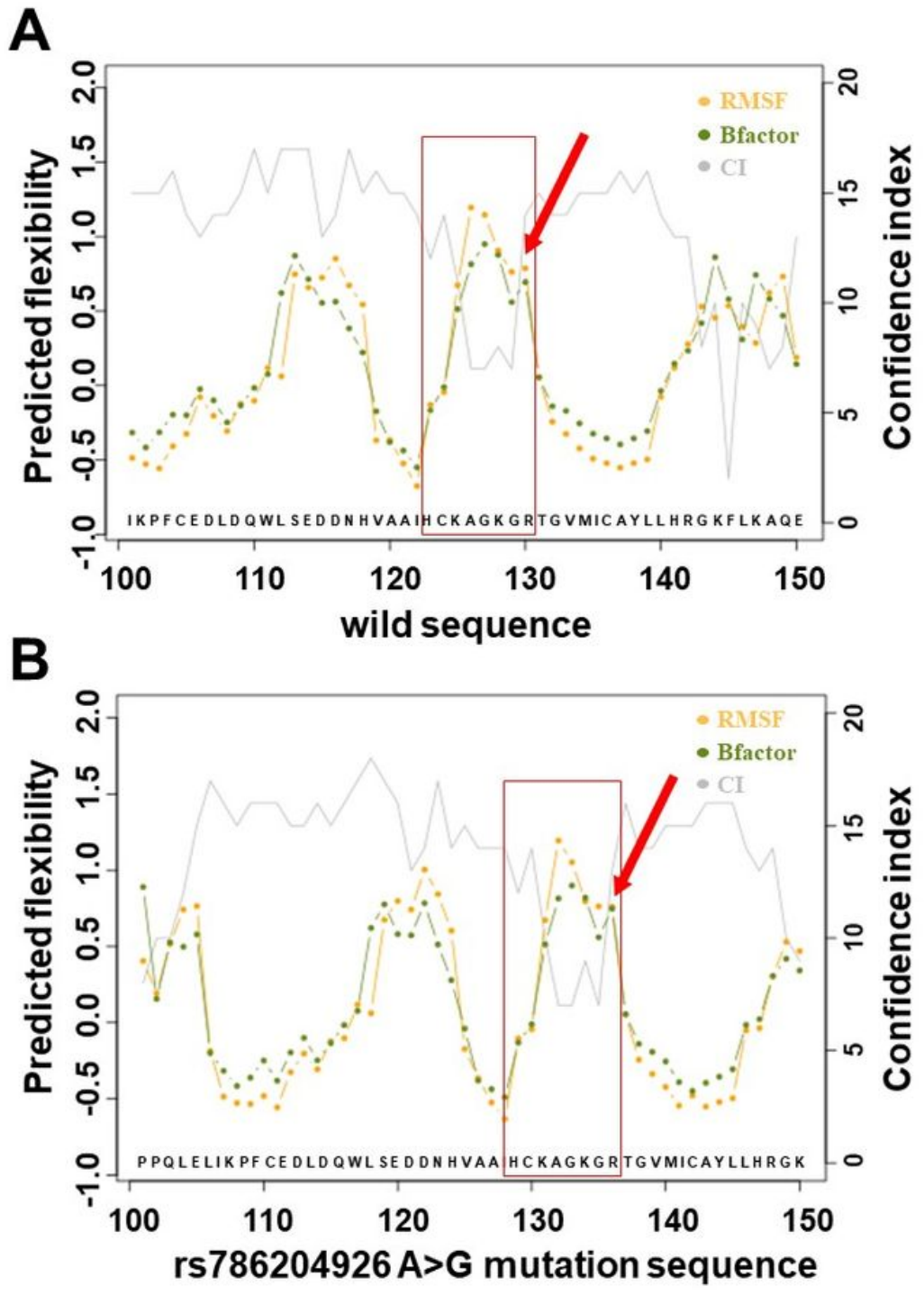

C

20

Wild: $\quad \cdots \cdot$ MTAIIKEIVS RNKRRYQEDG Mutation: …..MTAIIKEIVS RNKRRYQEDG

30 40

50

FDLDLTYIYP NIIAMGFPAE RLEGVYRNNI

FDLDLTYIYP NIIAMGFPAE RLEGVYRNNI $60 \quad 70 \quad 80$

DDVVRFLDSK HKNHYKIYNL CAERHYDTAK

DDVVRFLDSKK HKNHYKIYNL CAERHYDTAK

$90 \quad 100 \quad 110$

FNCRVAQYPF EDHNPPQLEL IKPFCEDLDQ

FNCRGYLFTT VAQYPFEDHN PPQLELIKPF

120

130

140

WLSEDDNHVA AIHCKAGKGR TGVMICAYLL

CEDLDQWLSE DDNHVAAIHC KAGKGRTGVM

$150 \quad 160 \quad 170$

HRGKFLKAQE ALDFYGEVRT RDKKGVTIPS

ICAYLLHRGK FLKAQEALDF YGEVRTRDKK

$180 \quad 190 \quad 200$

QRRYVYYYSY LLKNHLDYRP VALLFHKMMF

GVTIPSQRRY VYYYSYLLKN HLDYRPVALL

$$
210 \quad 220
$$

ETIIPMFSGGT CNPQFWCQL…...

FHKMMFETIIP MFSGGTCNPQ $\cdots \cdots .$.

Predicted flexibility class

0 rigid $\quad 1$ intermediate 2 flexible

Figure 6

Changes of protein flexibility after PTEN gene rs786204926A>G mutation. (A) Changes of the flexibility of the PTEN wild protein. (B) Changes of the flexibility of the PTEN mutation protein. The box part is the core area of phosphatase function, the arrow refers to the changed amino acid. (C) Flexibility changes in part of the PTEN protein. (The underline is the core area of phosphatase function)

\section{Supplementary Files}

This is a list of supplementary files associated with this preprint. Click to download.

- Additionalfile.docx 